\title{
On approximation of real numbers by real algebraic numbers
}

\author{
by
}

\author{
V. Beresnevich (Minsk)
}

1. Introduction. In this paper we consider several related problems of the theory of Diophantine approximation. The following notation will be used. We denote by $\# S$ the number of elements in a finite set $S$. The Lebesgue measure of a measurable set $S \subset \mathbb{R}$ is denoted by $|S|$. The set $S \subset \mathbb{R}$ has full measure means that $|\mathbb{R} \backslash S|=0$. Throughout the paper, $\Psi$ denotes a monotonic sequence of positive numbers. We denote by $P_{n}$ the set of integral polynomials of degree $\leq n$. The set of real algebraic numbers of degree $n$ is denoted by $\mathbb{A}_{n}$. Given a polynomial $P, H(P)$ denotes the height of $P$. Given an algebraic number $\alpha, H(\alpha)$ denotes the height of $\alpha$. We use the Vinogradov symbol $\ll$, which means " $\leq$ up to a constant multiplier". We begin with a short review.

In 1924 Khinchin proved a remarkable result on the approximation of real numbers by rationals [9]. According to his theorem, for almost all $x \in \mathbb{R}$ the inequality

$$
|q x-p|<\Psi(q)
$$

has at most finitely or infinitely many solutions $p, q \in \mathbb{Z}$ according as the sum $\sum_{q=1}^{\infty} \Psi(q)$ converges or diverges.

In $1932 \mathrm{~K}$. Mahler [13] introduced a classification of real numbers and showed [12] that almost all numbers are $S$-numbers. In fact, he proved that $w_{n}(x) \leq 4 n$ for almost all $x \in \mathbb{R}$, where $w_{n}(x)$ is defined to be the supremum of the set of real numbers $w$ for which the inequality

$$
|P(x)|<H(P)^{-w}
$$

has infinitely many solutions $P \in P_{n}$. At the same time Mahler conjectured that $w_{n}(x)=n$ for almost all $x \in \mathbb{R}$. In 1964 Mahler's conjecture was completely proved by V. Sprindžuk [15-17].

1991 Mathematics Subject Classification: 11J13, 11J83. 
There have been many efforts to make the error term more precise on the right hand side of inequality (1). The case $n=2$ has been individually considered by Cassels, Kubilius and Bernik. In 1966 A. Baker [1] proved that for almost all $x \in \mathbb{R}$ the inequality

$$
|P(x)|<\Psi^{n}(H(P))
$$

has at most finitely many solutions $P \in P_{n}$ if $\sum_{h=1}^{\infty} \Psi(h)<\infty$. At the same time Baker conjectured that a stronger result had to be true. Regarding this V. Bernik proved in 1989 [6] that for almost all $x \in \mathbb{R}$ the inequality

$$
|P(x)|<H(P)^{-n+1} \Psi(H(P))
$$

has at most finitely many solutions $P \in P_{n}$ if $\sum_{h=1}^{\infty} \Psi(h)<\infty$. There were some grounds to suppose that the convergence condition in Bernik's theorem could not be omitted. In this paper we confirm this by proving the following theorem.

THEOREM 1. Let $\Psi$ be a decreasing sequence of positive numbers such that $\sum_{h=1}^{\infty} \Psi(h)=\infty$. Then for almost all $x \in \mathbb{R}$ the inequality (3) has infinitely many solutions $P \in P_{n}$.

It should be noted that there is an analogous problem for polynomials of complex variables. One should expect that for almost all $z \in \mathbb{C}$ the inequality $|P(z)|<H(P)^{-(n-2) / 2} \Psi^{1 / 2}(H(P))$ has at most finitely or infinitely many solutions in integral polynomials of degree $\leq n$ according as $\sum_{h=1}^{\infty} \Psi(h)$ converges or diverges. The methods of this paper and those of [6] with necessary modifications can probably be applied for solving the problem. But the question remains open for both the convergence and the divergence case.

The ideas of this paper can also be generalized to Diophantine approximation of points of smooth manifolds. Consider the solubility problem for the inequality

$$
\left|a_{n} x_{n}+\ldots+a_{1} x_{1}+a_{0}\right|<H^{-n+1} \Psi(H),
$$

in $\left(a_{0}, \ldots, a_{n}\right) \in \mathbb{Z}^{n+1}$, where $H=\max \left\{\left|a_{0}\right|, \ldots,\left|a_{n}\right|\right\}$ and the points $\mathbf{x}=$ $\left(x_{1}, \ldots, x_{n}\right)$ lie on a manifold $M$. If $M=\mathbb{R}^{n}$, it has been shown by Groshev (see [18, pp. 28-33]) that a so-called Khinchin-type theorem is available. This means that for almost all $\mathbf{x} \in M$ the inequality (4) has at most finitely or infinitely many solutions according as $\sum_{h=1}^{\infty} \Psi(h)$ converges or diverges.

There have been many attempts to prove Khinchin-type theorems for manifolds of dimension $<n$ embedded in $\mathbb{R}^{n}$ satisfying various arithmetic, analytic and(or) geometric conditions. In particular, one is available when a manifold of dimension at least $\max \{2, n / 2\}$ satisfies a curvature condition that for surfaces in $\mathbb{R}^{3}$ corresponds to the Gaussian curvature being positive almost everywhere [8]. V. Bernik proved a Khinchin-type theorem for a 
manifold being a topological product of at least four 3-smooth curves in $\mathbb{R}^{2}$ with non-vanishing curvature almost everywhere [7]. A Khinchin-type theorem for inhomogeneous approximation by values of 2-degree integral polynomials has been obtained in [5].

Very recently, D. Y. Kleinbock and G. A. Margulis [10] have obtained a strong extremality result for general non-degenerate $C^{(l)}$-manifolds of dimension $d<n$ in $\mathbb{R}^{n}$. In addition they generalized the theorem of Baker (see (2)) to these manifolds; more precisely, they proved that the inequality (4) has infinitely many solutions almost nowhere provided that the decreasing sequence $h \Psi(h), h=1,2, \ldots$, satisfies $\sum_{h=1}^{\infty} h^{-1}(h \Psi(h))^{1 /(d l)}<\infty$. Generalizations to simultaneous approximation can also be considered.

In view of the existence of correlations between the approximation of zero by values of integral polynomials and approximation of real numbers by algebraic numbers, we are interested in the solubility of the inequality

$$
|x-\alpha|<H(\alpha)^{-n} \Psi(H(\alpha))
$$

in real algebraic numbers of degree $n$. It should be noted that there is a classification of Koksma for real numbers [11] based on the solubility of the inequality

$$
|x-\alpha|<H(\alpha)^{-w^{*}-1} .
$$

The error term in (6) is a particular case of that of (5). Koksma considered the value $w_{n}^{*}(x)$, which is defined to be the supremum of the set of real numbers $w^{*}$ such that the inequality (6) has infinitely many solutions in real algebraic numbers of degree $\leq n$, where $n \in \mathbb{N}$. It can be shown by the theorem of Sprindžuk [17], a result of Wirsing [19] and the lemma of Borel-Cantelli that $w_{n}^{*}(x)=n$ for almost all real $x$. We are interested in the measure of the set

$$
A_{n}(\Psi)=\left\{x \in \mathbb{R} \text { : inequality (5) holds for infinitely many } \alpha \in \mathbb{A}_{n}\right\} .
$$

It was expected that it would essentially depend on the behaviour of the $\sum_{h=1}^{\infty} \Psi(h)$ as in the polynomial case above. We prove

TheOrem 2. Let $\Psi$ be a decreasing sequence of positive numbers. Then

$$
\left|A_{n}(\Psi)\right|= \begin{cases}0 & \text { if } \sum_{h=1}^{\infty} \Psi(h)<\infty, \\ \text { full } & \text { if } \sum_{h=1}^{\infty} \Psi(h)=\infty .\end{cases}
$$

The proof of Theorem 2 is based on the distribution of real algebraic numbers. We use the concept of regular systems introduced by A. Baker and W. Schmidt [2].

Definition 1. Let $\Gamma$ be a countable set of real numbers and $N: \Gamma \rightarrow \mathbb{R}$ be a positive function. The pair $(\Gamma, N)$ is called a regular system if there exists a constant $C_{1}=C_{1}(\Gamma, N)>0$ such that for any interval $I$ there 
exists a sufficiently large number $T_{0}=T_{0}(\Gamma, N, I)>0$ such that for any integer $T \geq T_{0}$ there are $\alpha_{1}, \ldots, \alpha_{t}$ in $\Gamma \cap I$ such that

$$
\begin{gathered}
N\left(\alpha_{i}\right) \leq T \quad(1 \leq i \leq t), \\
\left|\alpha_{i}-\alpha_{j}\right| \geq T^{-1} \quad(1 \leq i<j \leq t), \\
t \geq C_{1}|I| T .
\end{gathered}
$$

For the set of algebraic numbers the function $N$ normally depends on the height of the corresponding algebraic number. A. Baker and W. Schmidt have found that the set of real algebraic numbers of degree $\leq n$ together with the function $N(\alpha)=H(\alpha)^{n+1}(\ln H(\alpha))^{-3 n^{2}}$ is a regular system. For $n=2$ this has been generalized by R. Baker [3] to the set of zeros of functions of a general form. Also, it has been shown in [4] that the set of quadratic irrationals on the interval $[0,1]$ together with the function $N(\alpha)=H(\alpha)^{3}$ is a regular system.

In this paper we extend this to the set of real algebraic numbers of any degree.

TheOREM 3. The set $\mathbb{A}_{n}$ together with the function

$$
N(\alpha)=\left(H(\alpha) /(1+|\alpha|)^{n}\right)^{n+1}
$$

is a regular system.

2. Effective measure bounds. Throughout this section, $n$ denotes an integer $\geq 2, Q$ a natural number, $\varepsilon$ a positive number, and $I$ an interval of the form $[a, b)$ embedded in $[-1 / 2,1 / 2)$. Given $n$ and $Q$, we define $P_{n}(Q)=$ $\left\{P \in P_{n}: H(P) \leq Q\right\}$. Given $I, Q, \varepsilon$ and $P \in P_{n}(Q)$, we denote by $\sigma(P)$ the set consisting of $x \in I$ satisfying

$$
|P(x)| \leq \varepsilon, \quad\left|P^{\prime}(x)\right| \geq 2|I|^{-1} .
$$

Given $n, I, Q$ and $\varepsilon$, let $B_{n, I}(Q, \varepsilon)$ denote the union of $\sigma(P)$ over all $P \in$ $P_{n}(Q)$. The aim of this section is to obtain an upper bound for $\left|B_{n, I}(Q, \varepsilon)\right|$. We use the following

Lemma 1. Given $n, I=[a, b), Q$ and $\varepsilon$ such that $\varepsilon<(16 Q)^{-1}$, define

$$
I_{\varepsilon}^{\prime \prime}=[a, a+\varepsilon] \cup[b-\varepsilon, b) \quad \text { and } \quad I_{\varepsilon}^{\prime}=I \backslash I_{\varepsilon}^{\prime \prime} .
$$

Then for any $P \in P_{n}(Q)$ such that $\sigma(P) \cap I_{\varepsilon}^{\prime} \neq \emptyset$, for any $x_{0} \in \sigma(P) \cap I_{\varepsilon}^{\prime}$ there exists $\alpha \in I$ such that $P(\alpha)=0,\left|P^{\prime}(\alpha)\right|>\left|P^{\prime}\left(x_{0}\right)\right| / 2$ and

$$
\left|x_{0}-\alpha\right|<2 \varepsilon\left|P^{\prime}(\alpha)\right|^{-1} \text {. }
$$

Proof. Let $P(x)=a_{n} x^{n}+\ldots+a_{0} \in P_{n}(Q)$ satisfy $\sigma(P) \cap I_{\varepsilon}^{\prime} \neq \emptyset$. Fix $x_{0} \in \sigma(P) \cap I_{\varepsilon}^{\prime}$. Given $x$ satisfying $\left|x-x_{0}\right| \leq \varepsilon$, we readily verify that $|x| \leq 1 / 2$. By Lagrange's formula, we have $P^{\prime}(x)=P^{\prime}\left(x_{0}\right)+P^{\prime \prime}\left(x_{1}\right)\left(x-x_{0}\right)$, 
where $x_{1}$ is a point between $x$ and $x_{0}$. Using $|x| \leq 1 / 2$, it is easy to obtain the estimate

$$
\left|P^{\prime \prime}\left(x_{1}\right)\right|=\left|n(n-1) a_{n} x_{1}^{n-2}+\ldots+2 a_{2}\right|<16 \max \left\{\left|a_{0}\right|, \ldots,\left|a_{n}\right|\right\} \leq 16 Q .
$$

Then

$$
\left|P^{\prime \prime}\left(x_{1}\right)\left(x-x_{0}\right)\right| \leq 16 Q \varepsilon<1 \leq|I|^{-1} \leq\left|P^{\prime}\left(x_{0}\right)\right| / 2 .
$$

Hence, for any $x$ satisfying $\left|x-x_{0}\right| \leq \varepsilon$, we have

$$
\left|P^{\prime}(x)\right| \geq\left|P^{\prime}\left(x_{0}\right)\right|-\left|P^{\prime \prime}\left(x_{1}\right)\left(x-x_{0}\right)\right|>\left|P^{\prime}\left(x_{0}\right)\right| / 2 .
$$

By Lagrange's formula we have $P(x)=P\left(x_{0}\right)+P^{\prime}\left(x_{2}\right)\left(x-x_{0}\right)$, where $x_{2}$ is between $x$ and $x_{0}$. As shown above, $\left|P^{\prime}\left(x_{2}\right)\right|>\left|P^{\prime}\left(x_{0}\right)\right| / 2$. Further, if we let $x=x_{0} \pm \varepsilon$ then $\left|P^{\prime}\left(x_{2}\right)\left(x-x_{0}\right)\right|>\varepsilon\left|P^{\prime}\left(x_{0}\right)\right| / 2 \geq \varepsilon$. Moreover, the expression $P^{\prime}\left(x_{2}\right)\left(x-x_{0}\right)$ has different signs at $x_{0}-\varepsilon$ and $x_{0}+\varepsilon$. Since $\left|P\left(x_{0}\right)\right| \leq \varepsilon$, we conclude that $P(x)=P\left(x_{0}\right)+P^{\prime}\left(x_{2}\right)\left(x-x_{0}\right)$ also has different signs at $x_{0} \pm \varepsilon$. Therefore, there exists $\alpha \in\left[x_{0}-\varepsilon, x_{0}+\varepsilon\right] \subset I$ satisfying $P(\alpha)=0$. As shown above, $\left|P^{\prime}(\alpha)\right| \geq\left|P^{\prime}\left(x_{0}\right)\right| / 2$. Next, by Taylor's formula, we write

$$
P\left(x_{0}\right)=\left(P^{\prime}(\alpha)+\frac{1}{2} P^{\prime \prime}\left(x_{3}\right)\left(x_{0}-\alpha\right)\right)\left(x_{0}-\alpha\right) .
$$

Using the estimate $\left|\frac{1}{2} P^{\prime \prime}\left(x_{3}\right)\left(x_{0}-\alpha\right)\right| \leq\left|P^{\prime}\left(x_{0}\right)\right| / 4$, we get

$$
\left|P^{\prime}(\alpha)+\frac{1}{2} P^{\prime \prime}\left(x_{3}\right)\left(x_{0}-\alpha\right)\right| \geq\left|P^{\prime}(\alpha)\right| / 2 \text {. }
$$

This inequality and $\left|P\left(x_{0}\right)\right| \leq \varepsilon$ yield (11). The proof is complete.

Proposition 1. Given $n$ and $I=[a, b)$, define $Q_{1}=\max \left\{\left(2^{n-1}|I|\right)^{-1 / n}\right.$, $\left.4 n^{2}\right\}$. Then $\left|B_{n, I}(Q, \varepsilon)\right| \leq n 2^{n+2} \varepsilon Q^{n}|I|$ for any $Q>Q_{1}$ and any $\varepsilon<$ $n^{-1} 2^{-n-2} Q^{-n}$.

Proof. Since the sets of solutions of the systems (10) defined by a polynomial $P$ and the polynomial $-P$ coincide, without loss of generality, we consider the polynomials of $P_{n}(Q)$ with the coefficient of $x^{n}$ being nonnegative. Given $P \in P_{n}(Q)$ and a real number $\alpha$ such that $P^{\prime}(\alpha) \neq 0$, $\sigma(P, \alpha)$ denotes the interval $\left\{x \in I:|x-\alpha|<2 \varepsilon\left|P^{\prime}(\alpha)\right|^{-1}\right\}$. Let $I_{\varepsilon}^{\prime}$ and $I_{\varepsilon}^{\prime \prime}$ be defined as in Lemma 1. For every $P \in P_{n}(Q)$, we define

$$
Z_{I}(P)=\left\{\alpha \in I: P(\alpha)=0 \text { and }\left|P^{\prime}(\alpha)\right| \geq|I|^{-1}\right\} .
$$

By Lemma 1 , for any $P \in P_{n}(Q)$ we have

$$
\sigma(P) \cap I_{\varepsilon}^{\prime} \subset \bigcup_{\alpha \in Z_{I}(P)} \sigma(P, \alpha) .
$$

Fix integers $a_{1}, \ldots, a_{n}$ such that $\left|a_{i}\right| \leq Q(i=1, \ldots, n)$ and $a_{n} \geq 0$. Set $R(x)=a_{n} x^{n}+\ldots+a_{1} x$ and $P_{n}(Q, R)=\left\{P \in P_{n}(Q): P-R \in \mathbb{Z}\right\}$. There exists a collection of pairwise non-intersecting intervals $\left[w_{i-1}, w_{i}\right) \subset I$ $(i=1, \ldots, s)$ which cover $I$ and the derivative $R^{\prime}$ is monotonic and of 
constant sign on each $\left[w_{i-1}, w_{i}\right)$. It is clear that $s$ can be chosen such that $1 \leq s \leq 2 n-2$. Order the set $Z_{I, R}=\bigcup_{P \in P_{n}(Q, R)} Z_{I}(P)$ as

$$
Z_{I, R}=\left\{\alpha_{1}^{(1)}, \ldots, \alpha_{1}^{\left(k_{1}\right)}, \alpha_{2}^{(1)}, \ldots, \alpha_{2}^{\left(k_{2}\right)}, \ldots, \alpha_{s}^{(1)}, \ldots, \alpha_{s}^{\left(k_{s}\right)}\right\} .
$$

Here $k_{i}=\#\left(Z_{I, R} \cap\left[w_{i-1}, w_{i}\right)\right)$ and $Z_{I, R} \cap\left[w_{i-1}, w_{i}\right)=\left\{\alpha_{i}^{(1)}, \ldots, \alpha_{i}^{\left(k_{i}\right)}\right\}$, where $\alpha_{i}^{(j)}<\alpha_{i}^{(j+1)}$. Given $P \in P_{n}(Q, R)$, by the identity $P^{\prime} \equiv R^{\prime}$, we have $\sigma(P, \alpha)=\sigma(R, \alpha)$ for any $\alpha \in Z_{I}(P)$. Using (12), we get

$$
\begin{aligned}
\left(\bigcup_{P \in P_{n}(Q, R)} \sigma(P)\right) \cap I_{\varepsilon}^{\prime} & \subset\left(\bigcup_{P \in P_{n}(Q, R)} \bigcup_{\alpha \in Z_{I}(P)} \sigma(R, \alpha)\right) \\
& =\bigcup_{i=1}^{s} \bigcup_{j=1}^{k_{i}} \sigma\left(R, \alpha_{i}^{(j)}\right) .
\end{aligned}
$$

Fix an index $i(1 \leq i \leq s)$. If $k_{i} \geq 2$ then we can consider two consecutive roots $\alpha_{i}^{(j)}$ and $\alpha_{i}^{(j+1)}$ of two polynomials $R+a_{0}^{i, j}$ and $R+a_{0}^{i, j+1}$ respectively. For convenience we assume that $R^{\prime}$ is increasing and positive on $\left[w_{i-1}, w_{i}\right)$. Then $R$ is monotonic on $\left[w_{i-1}, w_{i}\right)$, and $a_{0}^{i, j} \neq a_{0}^{i, j+1}$. It follows that $\left|a_{0}^{i, j}-a_{0}^{i, j+1}\right| \geq 1$. Using Lagrange's formula and the monotonicity of $R^{\prime}$, we get

$$
\begin{aligned}
1 & \leq\left|a_{0}^{i, j}-a_{0}^{i, j+1}\right|=\left|R\left(\alpha_{i}^{(j)}\right)-R\left(\alpha_{i}^{(j+1)}\right)\right| \\
& =\left|R^{\prime}\left(\widetilde{\alpha}_{i}^{(j)}\right)\right| \cdot\left|\alpha_{i}^{(j)}-\alpha_{i}^{(j+1)}\right| \leq\left|R^{\prime}\left(\alpha_{i}^{(j+1)}\right)\right| \cdot\left|\alpha_{i}^{(j)}-\alpha_{i}^{(j+1)}\right|,
\end{aligned}
$$

where $\widetilde{\alpha}_{i}^{(j)}$ is between $\alpha_{i}^{(j)}$ and $\alpha_{i}^{(j+1)}$. This implies $\left|R^{\prime}\left(\alpha_{i}^{(j+1)}\right)\right|^{-1} \leq \alpha_{i}^{(j+1)}$ $\alpha_{i}^{(j)}$, whence we readily get

$$
\sum_{j=1}^{k_{i}-1}\left|R^{\prime}\left(\alpha_{i}^{(j+1)}\right)\right|^{-1} \leq \sum_{j=1}^{k_{i}-1}\left(\alpha_{i}^{(j+1)}-\alpha_{i}^{(j)}\right)=\alpha_{i}^{\left(k_{i}\right)}-\alpha_{i}^{(1)} \leq w_{i}-w_{i-1} .
$$

The last inequality and $\left|R^{\prime}\left(\alpha_{i}^{(1)}\right)\right| \geq|I|^{-1}$ yield

$$
\sum_{j=1}^{k_{i}}\left|R^{\prime}\left(\alpha_{i}^{(j)}\right)\right|^{-1} \leq w_{i}-w_{i-1}+|I|
$$

This method can be applied to all situations, i.e. when the behaviour of $R^{\prime}$ differs from the above, giving (14). This estimate also remains true when $k_{i}=1$, and certainly when $k_{i}=0$. Summing (14) over all $i$, we find

$$
\sum_{i=1}^{s} \sum_{j=1}^{k_{i}}\left|R^{\prime}\left(\alpha_{i}^{(j)}\right)\right|^{-1} \leq \sum_{i=1}^{s}\left(w_{i}-w_{i-1}+|I|\right) \leq(2 n-1)|I| .
$$

The obvious estimate $|\sigma(R, \alpha)| \leq 4 \varepsilon\left|R^{\prime}(\alpha)\right|^{-1}$ together with (13) and (15) 
gives

$$
\left|\bigcup_{P \in P_{n}(Q, R)} \sigma(P) \cap I_{\varepsilon}^{\prime}\right| \leq 4 \varepsilon(2 n-1)|I| .
$$

We notice that

$$
B_{n, I}(Q, \varepsilon)=\bigcup_{R}\left(\bigcup_{P \in P_{n}(Q, R)} \sigma(P)\right) .
$$

Since the number of different polynomials $R$ is at most $(Q+1)(2 Q+1)^{n-1}$, using (16) and (17), we conclude that

$$
\left|B_{n, I}(Q, \varepsilon) \cap I_{\varepsilon}^{\prime}\right| \leq 4 \varepsilon(2 n-1)|I|(Q+1)(2 Q+1)^{n-1} .
$$

Now we make the following transformations:

$$
\begin{aligned}
\left|B_{n, I}(Q, \varepsilon)\right| \leq & 4 \varepsilon(2 n-1)|I|(Q+1)(2 Q+1)^{n-1}+2 \varepsilon \\
\leq & n 2^{n+2} \varepsilon Q^{n}|I|\left(\left(1-(2 n)^{-1}\right)\left(1+Q^{-1}\right)^{n}+2^{-n-1} n^{-1} Q^{-n}|I|^{-1}\right) \\
\leq & n 2^{n+2} \varepsilon Q^{n}|I| \\
& \times\left(\left(1-(4 n)^{-1}\right)\left(1+Q^{-1}\right)^{n}+2^{-n-1} n^{-1} Q^{-n}|I|^{-1}-(4 n)^{-1}\right) .
\end{aligned}
$$

The inequality $Q>\left(2^{n-1}|I|\right)^{-1 / n}$ gives $2^{-n-1} n^{-1} Q^{-n}|I|^{-1}-(4 n)^{-1}<0$. The inequality $Q>4 n^{2}$ implies $\left(1-(4 n)^{-1}\right)\left(1+Q^{-1}\right)^{n}<1$. Then we get the required estimate and the proof is complete.

3. Distribution of real algebraic numbers. This section is devoted to the study of the distribution of real algebraic numbers. To prove Theorem 3 we need the following

Proposition 2. Let I be a finite interval. Then for almost all $x \in I$ the system

$$
|P(x)|<H(P)^{-n}, \quad\left|P^{\prime}(x)\right|<2|I|^{-1}
$$

has at most finitely many solutions $P \in P_{n}$.

This follows from Propositions 1-3 of [6], where a more general statement is proved.

Now we proceed to prove Theorem 3. First of all, note that it is sufficient to show that the required distribution holds for any interval of length $\leq 1$. Fix an interval $I \subset[-1 / 2,1 / 2)$ and $Q \in \mathbb{N}$. Let $\varepsilon_{Q}=n^{-1} 2^{-n-5} Q^{-n}$. We now define five relatively small subsets of $I$.

1. The first is $B_{1}(I, Q)=B_{n, I}\left(Q, \varepsilon_{Q}\right)$. By Proposition 1, we have $\left|B_{1}(I, Q)\right| \leq|I| / 8$ whenever $Q>Q_{1}$. 
2. Given $P \in P_{n}$, define $\sigma_{2}(P)$ to consist of all solutions of system (18), and set

$$
B_{2}(I, Q)=\bigcup_{\substack{P \in P_{n} \\ H(P)>Q}} \sigma_{2}(P) .
$$

By Proposition 2, we have $\left|B_{2}(I, Q)\right| \rightarrow 0$ as $Q \rightarrow \infty$. Therefore, there exists a sufficiently large number $Q_{2}$ such that $\left|B_{2}\left(I, Q_{2}\right)\right| \leq|I| / 16$.

3. For any non-zero $P \in P_{n}\left(Q_{2}\right)$ define $\sigma_{3}(P, Q)=\left\{x \in I:|P(x)|<\varepsilon_{Q}\right\}$. Let $B_{3}\left(I, Q_{2}, Q\right)$ be the union of $\sigma_{3}(P, Q)$ over all $P \in P_{n}\left(Q_{2}\right) \backslash\{0\}$. Since $Q_{2}$ depends on $I$ and $n$ only, the number of different intervals $\sigma_{3}(P, Q)$ is bounded by a constant independent of $Q$. Moreover, $\left|\sigma_{3}(P, Q)\right| \rightarrow 0$ as $Q \rightarrow \infty$. Now, it is not difficult to see that there exists $Q_{3}>0$ such that for any $Q>Q_{3}$ we have $\left|B_{3}\left(I, Q_{2}, Q\right)\right| \leq|I| / 16$. Note that the constant $Q_{3}$ can be explicitly calculated.

4. We denote by $B_{4}(I, Q)$ the union of the intervals $\sigma_{4}(\alpha, Q)=\{x \in$ $\left.I:|x-\alpha| \leq 8 \varepsilon_{Q} Q^{-1}\right\}$ over all real algebraic numbers of degree $\leq n-1$ of height $\leq\left(n 2^{4 n+2}+1\right) Q$. Since the number of different intervals in this union is at most $\ll Q^{n}$ and every interval has length $\ll Q^{-(n+1)}$, there exists a sufficiently large number $Q_{4}>0$ such that $\left|B_{4}(I, Q)\right| \leq|I| / 8$ for any $Q>Q_{4}$. The constant $Q_{4}$ can be explicitly calculated.

5. Finally, set $B_{5}(I, Q)=\left[a, a+\varepsilon_{Q}\right] \cup\left[b-\varepsilon_{Q}, b\right]$. Whenever $Q>Q_{5}=$ $\left(|I| n 2^{n+1}\right)^{-1 / n}$, we have $\left|B_{5}(I, Q)\right| \leq|I| / 8$.

Now we define

$$
B(I, Q)=B_{1}(I, Q) \cup B_{2}\left(I, Q_{2}\right) \cup B_{3}\left(I, Q_{2}, Q\right) \cup B_{4}(I, Q) \cup B_{5}(I, Q) .
$$

According to our calculations above, whenever $Q>\max \left\{Q_{1}, \ldots, Q_{5}\right\}$ we have the estimate $|B(I, Q)| \leq|I| / 2$.

Let $x \in I \backslash B(I, Q)$. By Minkowski's linear forms theorem [14, Ch. 2, §3], there exists a non-zero polynomial $P(t)=a_{n} t^{n}+\ldots+a_{0} \in P_{n}$ satisfying

$$
|P(x)| \leq \varepsilon_{Q}, \quad\left|P^{\prime}(x)\right| \leq n 2^{4 n+2} Q, \quad\left|a_{i}\right| \leq Q / 8 \quad(2 \leq i \leq n) .
$$

Assume that $\left|P^{\prime}(x)\right| \leq Q / 2$. Then, using $|x| \leq 1 / 2$ and (19), we find

$$
\left|a_{1}\right| \leq\left|P^{\prime}(x)\right|+\sum_{i=2}^{n}\left|i a_{i} x^{i-1}\right| \leq \frac{Q}{2}+\frac{Q}{8} \sum_{i=2}^{n} i 2^{-i+1} \leq Q .
$$

Next, (19) together with $|x| \leq 1 / 2$ gives

$$
\left|a_{0}\right| \leq|P(x)|+\sum_{i=1}^{n}\left|a_{i} x^{i}\right| \leq \frac{Q}{2}+Q \sum_{i=1}^{\infty} 2^{-i} \leq Q .
$$

It follows that $H(P) \leq Q$. It is now easy to see that $x$ belongs to one of the sets $B_{1}(I, Q), B_{2}\left(I, Q_{2}\right)$ or $B_{3}\left(I, Q_{2}, Q\right)$, contrary to $x$ being a point of 
$I \backslash B(I, Q)$. Hence, whenever $x \in I \backslash B(Q, I)$, there exists a non-zero solution $P \in P_{n}$ of the system (19) such that

$$
\left|P^{\prime}(x)\right|>Q / 2 \text {. }
$$

Using (19), it is easy to obtain by the same method as above that

$$
H(P) \leq\left(n 2^{4 n+2}+1\right) Q .
$$

Now we are going to show that there exists a root of $P$ very close to $x$. To this end we define the constants $Q_{6}=2^{(3 n-3) /(n-1)}+1$ and $Q_{7}=4|I|^{-1}$ and ensure that $Q>Q_{0}=\max \left\{Q_{1}, \ldots, Q_{7}\right\}$. In this situation we can apply Lemma 1, and conclude that there exists a real root $\alpha$ of $P$ in $I$ such that

$$
|x-\alpha| \leq \frac{2|P(x)|}{\left|P^{\prime}(x)\right| / 2} \leq 8 \varepsilon_{Q} Q^{-1} .
$$

By $(21)$, we have $H(\alpha) \leq\left(n 2^{4 n+2}+1\right) Q$. Since $x \notin B(I, Q)$, we have $x \notin B_{4}(I, Q)$. It follows that the degree of $\alpha$ is exactly $n$.

We choose a maximal collection $\left\{\alpha_{1}, \ldots, \alpha_{t}\right\} \subset I$ consisting of real algebraic numbers with $\operatorname{deg} \alpha_{i}=n$,

$$
H\left(\alpha_{i}\right) \leq\left(n 2^{4 n+2}+1\right) Q
$$

for all $i \in\{1, \ldots, t\}$ and

$$
\left|\alpha_{i}-\alpha_{j}\right| \geq 8 \varepsilon_{Q} Q^{-1} \quad(1 \leq i<j \leq t) .
$$

As we have proved, for any $x \in I \backslash B(I, Q)$ there exists $\alpha \in \mathbb{A}_{n}$ satisfying $H(\alpha) \leq\left(n 2^{4 n+2}+1\right) Q$ and $(22)$. Since the collection $\left\{\alpha_{1}, \ldots, \alpha_{t}\right\}$ is maximal, there exists $\alpha_{i}$ in this collection such that $\left|\alpha-\alpha_{i}\right| \leq 8 \varepsilon_{Q} Q^{-1}$. Hence, $\left|x-\alpha_{i}\right| \leq 16 \varepsilon_{Q} Q^{-1}$ and so

$$
I \backslash B(I, Q) \subset \bigcup_{i=1}^{t}\left\{x \in I:\left|x-\alpha_{i}\right| \leq 16 \varepsilon_{Q} Q^{-1}\right\} .
$$

Since $|I \backslash B(I, Q)| \geq|I| / 2$ we have $|I| / 2 \leq|I \backslash B(I, Q)| \leq t \cdot 32 \varepsilon_{Q} Q^{-1}$ and we get

$$
t \geq n 2^{n-1} Q^{n+1}|I| .
$$

Now, let $J$ be any interval of length $\leq 1$. There exists an integer $m$ such that $J_{m}=(J+m) \cap[-1 / 2,1 / 2)$ has measure $\geq|J| / 2$. Let $Q_{0}\left(J_{m}\right)=$ $\max \left\{Q_{1}\left(J_{m}\right), \ldots, Q_{7}\left(J_{m}\right)\right\}$, where $Q_{1}, \ldots, Q_{7}$ are defined as above. As we have proved, for any $Q>Q_{0}\left(J_{m}\right)$ there is a collection $\left\{\alpha_{1}, \ldots, \alpha_{t}\right\} \subset \mathbb{A}_{n} \cap J_{m}$ satisfying (23)-(25). The numbers $\beta_{i}=\alpha_{i}-m \in J$ are algebraic of degree $n$ as well. If $m \neq 0$ one readily verifies that $H\left(\beta_{i}\right) \leq H\left(\alpha_{i}\right)(1+|m|)^{n+1} /|m|$. Then, using this together with $(23)$ and the obvious inequality $1+|m| \leq$ $2\left(1+\left|\beta_{i}\right|\right)$, we get $H\left(\beta_{i}\right) \leq\left(1+\left|\beta_{i}\right|\right)^{n} n 2^{5 n+4} Q$. Writing this in terms of the 
function $N$, we get

$$
N\left(\beta_{i}\right) \leq\left(n 2^{5 n+4}\right)^{n+1} Q^{n+1} .
$$

The last inequality also holds when $m=0$. Further, by (24), (25) and $|J| \geq\left|J_{m}\right| / 2$, we have

$$
\begin{gathered}
\left|\beta_{i}-\beta_{j}\right| \geq n^{-1} 2^{-n-2} Q^{-n-1} \quad(1 \leq i<j \leq t), \\
t \geq n 2^{n-2} Q^{n+1}|J| .
\end{gathered}
$$

Now let $T_{0}=\left(n 2^{5 n+4}\right)^{n+1}\left(Q_{0}\left(J_{m}\right)+1\right)^{n+1}$. Then for any integer $T>$ $T_{0}$ the number $Q=\left[T^{1 /(n+1)} /\left(n 2^{5 n+4}\right)\right]$ is greater than $Q_{0}\left(J_{m}\right)$. As we have shown, there exist $\beta_{1}, \ldots, \beta_{t} \in J \cap \mathbb{A}_{n}$ satisfying (26)-(28). Then we correspondingly have

$$
\begin{gathered}
N\left(\beta_{i}\right) \leq T \quad(1 \leq i \leq t), \\
\left|\beta_{i}-\beta_{j}\right| \geq n^{-1} 2^{-n-2}\left(n 2^{5 n+4}\right)^{n+1} T^{-1}>T^{-1} \quad(1 \leq i<j \leq t), \\
t \geq n 2^{n-2}\left(n 2^{5 n+5}\right)^{-n-1} T|J|=\left(n^{n} 2^{5 n^{2}+9 n+7}\right)^{-1} T|J| .
\end{gathered}
$$

This completes the proof of Theorem 3 with $C_{1}=\left(n^{n} 2^{5 n^{2}+9 n+7}\right)^{-1}$.

4. Proof of Theorem 2. We proceed to prove Theorem 2. For any $\alpha \in \mathbb{A}_{n}$ we define the interval

$$
\sigma(\alpha)=\left\{x \in \mathbb{R}:|x-\alpha|<H(\alpha)^{-n} \Psi(H(\alpha))\right\} .
$$

It is easy to see that the set $A_{n}(\Psi)$ consists of all $x \in \mathbb{R}$ belonging to infinitely many intervals $\sigma(\alpha)$.

First we consider the convergence part of Theorem 2. The following calculation is readily verified:

$$
\sum_{\alpha \in \mathbb{A}_{n}}|\sigma(\alpha)|=\sum_{h=1}^{\infty} \sum_{\substack{\alpha \in \mathbb{A}_{n} \\ H(\alpha)=h}}|\sigma(\alpha)|=\sum_{h=1}^{\infty} \sum_{\substack{\alpha \in \mathbb{A}_{n} \\ H(\alpha)=h}} 2 h^{-n} \Psi(h) \ll \sum_{h=1}^{\infty} \Psi(h)<\infty .
$$

The Borel-Cantelli Lemma finishes the proof.

Now we proceed to prove the divergence part. We use the following lemmas.

Lemma 2. Let $A$ be a measurable set. If there is a positive constant $C_{2}<1$ such that $|A \cap I| \geq C_{2}|I|$ for any finite interval $I \subset \mathbb{R}$, then $A$ has full measure.

Proof. Suppose that $|\mathbb{R} \backslash A|>0$. Then, by the Lebesgue measure density theorem, there exists $x_{0} \in \mathbb{R}$ such that for any $0<\varepsilon<1$ there is $\delta>0$ such that $\left|(\mathbb{R} \backslash A) \cap\left[x_{0}-\delta, x_{0}+\delta\right]\right| \geq 2 \delta(1-\varepsilon)$. It follows that $\left|A \cap\left[x_{0}-\delta, x_{0}+\delta\right]\right|<2 \delta(1-\varepsilon)$. Putting $\varepsilon=1-C_{2}$, we obtain a contradiction. The proof is finished. 
Lemma 3. Let $E_{i} \subset \mathbb{R}$ be a sequence of measurable sets and let the set $E$ consist of the points belonging to infinitely many $E_{i}$. If all the sets $E_{i}$ are totally bounded and $\sum_{i=1}^{\infty}\left|E_{i}\right|$ diverges, then

$$
|E| \geq \limsup _{N \rightarrow \infty} \frac{\left(\sum_{i=1}^{N}\left|E_{i}\right|\right)^{2}}{\sum_{i=1}^{N} \sum_{j=1}^{N}\left|E_{i} \cap E_{j}\right|} .
$$

This lemma is proved in [18, Chapter $2, \S 2]$.

LEMmA 4. Let $\left\{a_{i}\right\}_{i=1}^{\infty}$ be a decreasing sequence of positive numbers such that $\sum_{i=1}^{\infty} a_{i}$ diverges. Define $b_{i}=\min \left\{a_{i} ; i^{-1}\right\}$. Then $\left\{b_{i}\right\}_{i=1}^{\infty}$ is also decreasing and $\sum_{i=1}^{\infty} b_{i}$ diverges.

Proof. The monotonicity of $b_{i}$ is readily verified. Further, assume that $\sum_{i=1}^{\infty} b_{i}$ converges. It follows that

$$
a_{i}>i^{-1} \quad \text { for infinitely many } i \text {. }
$$

Since $b_{i}$ is monotonic, for any integer $l>3$ we have $\sum_{i=[l / 2]}^{l} b_{i} \geq \sum_{i=[l / 2]}^{l} b_{l}$ $\geq l b_{l} / 2$, which implies

$$
l b_{l} \leq 2 \sum_{i=[l / 2]}^{l} b_{i} .
$$

Since we have assumed that $\sum_{i=1}^{\infty} b_{i}<\infty$, we infer $\sum_{i=[l / 2]}^{l} b_{i} \rightarrow 0$ as $l \rightarrow \infty$. By (30), we get $l b_{l} \rightarrow 0$ as $l \rightarrow \infty$. But according to (29) and the definition of $b_{i}$ we have $b_{i} \geq 1$ infinitely often. The derived contradiction tells us that the series $\sum_{i=1}^{\infty} b_{i}$ must diverge. The proof is complete.

LEMMA 5. Given a decreasing sequence $\Psi$ of positive numbers such that $\sum_{h=1}^{\infty} \Psi(h)$ converges (diverges), for any number $c>0$ the series $\sum_{k=0}^{\infty} 2^{k} \Psi\left(c 2^{k}\right)$ converges (diverges).

Lemma 5 follows from the corresponding property of the integral

$$
\int_{1}^{\infty} 2^{x} \widetilde{\Psi}\left(c 2^{x}\right) d x=\frac{1}{c \log 2} \int_{1}^{\infty} \widetilde{\Psi}(y) d y
$$

where $\widetilde{\Psi}$ is monotonic and continuous on $\{x: x \geq 1\}$ and coincides with $\Psi$ on $\mathbb{N}$.

Now we are ready to prove Theorem 2. Fix any finite interval $I \subset \mathbb{R}$ and set $r=(1+\sup \{|x|: x \in I\})^{n}$. Define $\Psi_{0}(h)=\min \left\{\Psi(h), h^{-1} / 2\right\}$. By Lemma 4 , this sequence is monotonic and $\sum_{h=1}^{\infty} \Psi_{0}(h)$ diverges. Moreover, by the definition we have

$$
h^{-n} \Psi_{0}(h) \leq h^{-n-1} / 2 \quad \text { for all } h \in \mathbb{N} .
$$


Define $\Phi(h)=h \Psi_{0}(h) / r^{n+1}$. Then Lemma 5 implies

$$
\sum_{k=0}^{\infty} \Phi\left(r 2^{k}\right)=\infty
$$

By Theorem 3, there exist positive constants $C_{1}=C_{1}(n)$ and $k_{0}=$ $k_{0}(n, I)$ such that for any $k \geq k_{0}$ there is a collection

$$
A_{k}(I)=\left\{\alpha_{1}<\ldots<\alpha_{t_{k}}\right\} \subset \mathbb{A}_{n} \cap I
$$

satisfying the following conditions:

(34) $|\alpha-\beta| \geq 2^{-k(n+1)} \quad$ for any numbers $\alpha, \beta \in A_{k}(I)$, with $\alpha \neq \beta$,

$$
C_{1} 2^{(n+1) k}|I| \leq t_{k} \leq 2^{(n+1) k}|I| .
$$

These conditions correspond to (7)-(9). Define

$$
\begin{aligned}
E_{k}(\alpha) & =\left\{x \in \mathbb{R}:|x-\alpha|<\left(r 2^{k}\right)^{-n} \Psi_{0}\left(r 2^{k}\right)\right\} \quad\left(\alpha \in A_{k}(I)\right), \\
E_{k} & =\bigcup_{\alpha \in A_{k}(I)} E_{k}(\alpha) .
\end{aligned}
$$

It is easy to verify that

$$
\left|E_{k}(\alpha)\right|=2\left(r 2^{k}\right)^{-n} \Psi_{0}\left(r 2^{k}\right)=2 \cdot 2^{-k(n+1)} \Phi\left(r 2^{k}\right) .
$$

Set

$$
E(I)=\bigcap_{N=k_{0}}^{\infty} \bigcup_{k=N}^{\infty} E_{k} .
$$

Since $\Psi$ is monotonic and $\Psi_{0}(h) \leq \Psi(h)$ for all $h \in \mathbb{N}$, by (33), we have $E_{k}(\alpha) \subset \sigma(\alpha)$. It follows that $E(I) \subset A_{n}(\Psi)$. Since $\left|E_{k}(\alpha)\right| \rightarrow 0$ as $k \rightarrow \infty$ and $A_{k}(I) \subset I$, we have $E(I) \subset \bar{I}$, where $\bar{I}$ is the topological closure of $I$. Then $E(I) \subset A_{n}(\Psi) \cap \bar{I}$. Since the boundary of $I$ evidently has zero measure, we conclude that

$$
\left|A_{n}(\Psi) \cap I\right| \geq|E(I)| .
$$

By (31) and (34), $E_{k}(\alpha) \cap E_{k}(\beta)=\emptyset$ for all $\alpha, \beta \in A_{k}(I), \alpha \neq \beta$. Then $\left|E_{k}\right|=t_{k} \cdot\left|E_{k}(\alpha)\right|$, where $\alpha \in A_{k}(I)$. By (35) and (36), we have

$$
2 C_{1} \Phi\left(r 2^{k}\right)|I| \leq\left|E_{k}\right| \leq 2 \Phi\left(r 2^{k}\right)|I| \text {. }
$$

It follows that

$$
\sum_{k=k_{0}}^{N}\left|E_{k}\right| \geq 2 C_{1}|I| \sum_{k=k_{0}}^{N} \Phi\left(r 2^{k}\right) .
$$


Using (32) and (39), we get

$$
\sum_{k=k_{0}}^{\infty}\left|E_{k}\right|=\infty
$$

We proceed to estimate the measures of the intersections. Fix, as we may by (32), a number $N_{0}>k_{0}$ such that

$$
\sum_{k=k_{0}}^{N_{0}} \Phi\left(r 2^{k}\right)>1
$$

Fix $k$ and $l$ such that $k_{0} \leq k<l \leq N$, where $N>N_{0}$. For any $\alpha \in A_{k}(I)$ we have

$$
E_{l} \cap E_{k}(\alpha)=\bigcup_{\beta \in A_{l}(I)} E_{k}(\beta) \cap E_{k}(\alpha) .
$$

Given $\alpha \in A_{k}(I)$, the number of different $\beta \in A_{l}(I)$ satisfying $E_{l}(\beta) \cap$ $E_{k}(\alpha) \neq \emptyset$ is less than

$$
2+\left|E_{k}(\alpha)\right| / 2^{-(n+1) l} \stackrel{(36)}{=} 2+2 \cdot 2^{(n+1)(l-k)} \Phi\left(r 2^{k}\right) .
$$

Using (36) and (41), we get

$$
\begin{aligned}
\left|E_{l} \cap E_{k}(\alpha)\right| & \leq \max _{\beta \in A_{l}(I)}\left\{\left|E_{l}(\beta)\right|\right\}\left(2+2 \cdot 2^{(n+1)(l-k)} \Phi\left(r 2^{k}\right)\right) \\
& \leq 4 \cdot 2^{-(n+1) l} \Phi\left(r 2^{l}\right)\left(1+2^{(n+1)(l-k)} \Phi\left(r 2^{k}\right)\right) .
\end{aligned}
$$

This is used in the following calculations:

$$
\begin{aligned}
\left|E_{l} \cap E_{k}\right| & \leq t_{k} \cdot \max _{\alpha \in A_{k}(I)}\left\{\left|E_{l} \cap E_{k}(\alpha)\right|\right\} \\
& \leq 4 t_{k} 2^{-(n+1) l} \Phi\left(r 2^{l}\right)\left(1+2^{(n+1)(l-k)} \Phi\left(r 2^{k}\right)\right) \\
& \stackrel{(35)}{\leq} 4|I| \Phi\left(r 2^{l}\right) \Phi\left(r 2^{k}\right)+4|I| 2^{-(n+1)(l-k)} \Phi\left(r 2^{l}\right) .
\end{aligned}
$$

Since $E_{k} \cap E_{l}=E_{l} \cap E_{k}$ we have

$$
\sum_{l=k_{0}}^{N} \sum_{k=k_{0}}^{N}\left|E_{l} \cap E_{k}\right|=\sum_{k=k_{0}}^{N}\left|E_{k}\right|+2 \sum_{l=k_{0}+1}^{N} \sum_{k=k_{0}}^{l-1}\left|E_{l} \cap E_{k}\right| .
$$

By (38), we get

$$
\sum_{k=k_{0}}^{N}\left|E_{k}\right| \leq 2|I| \sum_{k=k_{0}}^{N} \Phi\left(r 2^{k}\right) .
$$

The second summand from (43) is estimated with the help of (42): 


$$
\begin{aligned}
2 \sum_{l=k_{0}+1}^{N} \sum_{k=k_{0}}^{l-1}\left|E_{l} \cap E_{k}\right| \leq & 8|I| \sum_{l=k_{0}+1}^{N} \sum_{k=k_{0}}^{l-1} \Phi\left(r 2^{l}\right) \Phi\left(r 2^{k}\right) \\
& +8|I| \sum_{l=k_{0}+1}^{N} \sum_{k=k_{0}}^{l-1} 2^{-(n+1)(l-k)} \Phi\left(r 2^{l}\right) .
\end{aligned}
$$

We transform the last term as follows:

$$
\begin{aligned}
& 8|I| \sum_{l=k_{0}+1}^{N} \sum_{k=k_{0}}^{l-1} 2^{-(n+1)(l-k)} \Phi\left(r 2^{l}\right) \\
& \quad=8|I| \sum_{l=k_{0}+1}^{N} \Phi\left(r 2^{l}\right) \sum_{k=k_{0}}^{l-1} 2^{-(n+1)(l-k)} \leq 2|I| \sum_{l=k_{0}+1}^{N} \Phi\left(r 2^{l}\right) .
\end{aligned}
$$

By (40) and (43)-(46), we conclude that

$$
\begin{aligned}
\sum_{l=k_{0}}^{N} \sum_{k=k_{0}}^{N}\left|E_{l} \cap E_{k}\right| & \leq 4|I| \sum_{k=k_{0}}^{N} \Phi\left(r 2^{k}\right)+8|I| \sum_{l=k_{0}+1}^{N} \sum_{k=k_{0}}^{l-1} \Phi\left(r 2^{l}\right) \Phi\left(r 2^{k}\right) \\
& \leq 4|I|\left(\sum_{k=k_{0}}^{N} \Phi\left(r 2^{k}\right)\right)^{2}+4|I| \sum_{l=k_{0}}^{N} \sum_{k=k_{0}}^{N} \Phi\left(r 2^{l}\right) \Phi\left(r 2^{k}\right) \\
& =8|I|\left(\sum_{k=k_{0}}^{N} \Phi\left(r 2^{k}\right)\right)^{2} .
\end{aligned}
$$

This estimate and (39) give

$$
\frac{\left(\sum_{k=k_{0}}^{N}\left|E_{k}\right|\right)^{2}}{\left(\sum_{k=k_{0}}^{N} \sum_{l=k_{0}}^{N}\left|E_{k} \cap E_{l}\right|\right)} \geq \frac{\left(2 C_{1}|I|\right)^{2}\left(\sum_{k=k_{0}}^{N} \Phi\left(r 2^{k}\right)\right)^{2}}{8|I|\left(\sum_{k=k_{0}}^{N} \Phi\left(r 2^{k}\right)\right)^{2}}=C_{1}^{2}|I| / 2,
$$

for any $N>N_{0}$. The conditions of Lemma 3 are satisfied. It follows that $|E(I)| \geq C_{1}^{2}|I| / 2$. By (37), we get $|A(\Psi) \cap I| \geq C_{1}^{2}|I| / 2$. This holds for any finite interval $I$. Lemma 2 completes the proof of Theorem 2.

5. Proof of Theorem 1. Now we are ready to give the proof of Theorem 1. Let $P_{n}(\Psi)$ denote the set of real numbers $x$ satisfying the inequality (3) for infinitely many polynomials $P \in P_{n}$. Fix a constant $r>0$. Given $d>0$, define $\Psi_{d}(h)=\Psi(h) / d$. It is clear that $\Psi_{d}(h)$ is monotonic and $\sum_{h=1}^{\infty} \Psi_{d}(h)$ diverges. By Theorem 2 , the set $A_{n}\left(\Psi_{d}\right)$ has full measure. It follows that $\mu\left(A_{n}\left(\Psi_{d}\right) \cap[-r, r]\right)=2 r$. Given $\alpha \in \mathbb{A}_{n}$, define $\sigma_{r, d}(\alpha)=\left\{x \in[-r, r]:|x-\alpha|<H(\alpha)^{-n} \Psi_{d}(H(\alpha))\right\}$. Then

$$
A_{n}\left(\Psi_{d}\right) \cap[-r, r]=\bigcap_{k=1}^{\infty} \bigcup_{\alpha: H(\alpha)>k} \sigma_{r, d}(\alpha) .
$$


Given $\alpha \in \mathbb{A}_{n}$, denote the minimal polynomial of $\alpha$ by $P_{\alpha}$. It can be written in the form

$$
P_{\alpha}(t)=(t-\alpha) \sum_{k=1}^{n} \frac{1}{k !} P_{\alpha}^{(k)}(\alpha)(t-\alpha)^{k-1} .
$$

Fix $x \in \sigma_{r, d}(\alpha)$. Since $x \in[-r, r]$ and $|\alpha-x|<H(\alpha)^{-n} \Psi_{d}(H(\alpha))$, we get

$$
\left|\sum_{k=1}^{n} \frac{1}{k !} P_{\alpha}^{(k)}(\alpha)(x-\alpha)^{k-1}\right| \leq C_{3} H(\alpha),
$$

where $C_{3}=C_{3}(r)$ is a constant. Now we readily get the estimate

$$
\left|P_{\alpha}(x)\right| \leq C_{3}|x-\alpha| H(\alpha) .
$$

Let $d=C_{3}$. Then for any $\alpha \in \mathbb{A}_{n}$ such that $\sigma_{r, C_{3}}(\alpha) \neq \emptyset$ and any $x \in \sigma_{r, C_{3}}(\alpha)$ we have

$$
\begin{aligned}
\left|P_{\alpha}(x)\right| & \leq C_{3} H(\alpha)|x-\alpha|<C_{3} H(\alpha) H(\alpha)^{-n} \Psi_{C_{3}}(H(\alpha)) \\
& =H\left(P_{\alpha}\right)^{-n+1} \Psi\left(H\left(P_{\alpha}\right)\right) .
\end{aligned}
$$

Thus, if $x \in \sigma_{r, C_{3}}(\alpha)$ then $P_{\alpha}$ is a solution of (3). It follows that if $x \in$ $A_{n}\left(\Psi_{C_{3}}\right)$ then (3) has infinitely many solutions, and $x \in P_{n}(\Psi)$. Thus,

$$
A_{n}\left(\Psi_{C_{3}}\right) \cap[-r, r] \subset P_{n}(\Psi) \cap[-r, r] .
$$

It follows that $\left|P_{n}(\Psi) \cap[-r, r]\right|=2 r$ for any $r>0$. This means that $P_{n}(\Psi)$ has full measure. The proof is complete.

Acknowledgments. The author wishes to express his thanks to Prof. V. Bernik for suggesting the problem and for many stimulating conversations. The author gratefully acknowledges the helpful suggestions of Prof. V. Bernik, Prof. M. Dodson and Dr. D. Dickinson during the seminar devoted to the results of this paper in York University.

\section{References}

[1] A. Baker, On a theorem of Sprindžuk, Proc. Roy. Soc. London Ser. A 292 (1966), 92-104.

[2] A. Baker and W. Schmidt, Diophantine approximation and Hausdorff dimension, Proc. London Math. Soc. 21 (1970), 1-11.

[3] R. Baker, Sprindžuk's theorem and Hausdorff dimension, Mathematika 23 (1976), 184-196.

[4] V. Beresnevich, Effective measure estimates for sets of real numbers with a given error of approximation by quadratic irrationalities, Vestsi Akad. Navuk Belarusi Ser. Fiz. Mat. Navuk 1996, no. 4, 10-15 (in Russian).

[5] V. Beresnevich, V. Bernik and M. Dodson, Inhomogeneous non-linear Diophantine approximation, in: Papers in honor of Sprindžuk's 60th birthday, Minsk, 1997, 13-20. 
[6] V. Bernik, On the best approximation of zero by values of integral polynomials, Acta Arith. 53 (1989), 17-28 (in Russian).

[7] - Asymptotic behavior of the number of solutions for some systems of inequalities in the theory of Diophantine approximation of dependent quantities, Vestsi Akad. Navuk BSSR Ser. Fiz. Mat. Navuk 1973, no. 1, 10-17 (in Russian).

[8] M. M. Dodson, B. P. Rynne and J. A. G. Vickers, Khinchine-type theorems on manifolds, Acta Arith. 57 (1991), 115-130.

[9] A. Khintchine [A. Khinchin], Continued Fractions, University of Chicago Press, 1964.

[10] D. Y. Kleinbock and G. A. Margulis, Flows on homogeneous spaces and Diophantine approximation on manifolds, preprint 97-108, SF 343 Diskrete Strukturen in der Mathematik, Universität Bielefeld.

[11] J. Koksma, Über die Mahlersche Klasseneinteilung der transzendenten Zahlen und die Approximation komplexer Zahlen durch algebraische Zahlen, Monatsh. Math. Phys. 48 (1939), 176-189.

[12] K. Mahler, Über das Maß der Menge aller S-Zahlen, Math. Ann. 106 (1932), 131-139.

[13] —, Zur Approximation der Exponentialfunktion und des Logarithmus, Teil I, J. Reine Angew. Math. 166 (1932), 118-150.

[14] W. Schmidt, Diophantine Approximation, Springer, Berlin, 1980.

[15] V. Sprindžuk, More on Mahler's conjecture, Soviet Math. Dokl. 5 (1964), 361-363.

[16] - , The proof of Mahler's conjecture on the measure of the set of S-numbers, Izv. Akad. Nauk SSSR Math. Series 19 (1965), 191-194 (in Russian).

[17] —, Mahler's Problem in the Metric Theory of Numbers, Transl. Math. Monographs 25, Amer. Math. Soc., Providence, R.I., 1969.

[18] - Metric Theory of Diophantine Approximation, Wiley, New York, 1979.

[19] E. Wirsing, Approximation mit algebraischen Zahlen beschränkten Grades, J. Reine Angew. Math. 206 (1961), 67-77.

Institute of Mathematics

Academy of Sciences of Belarus

220072, Surganova 11, Minsk, Belarus

E-mail: beresnevich@im.bas-net.by 